\title{
HÍBRIDOS DOBLES DE MAÍZ FORMADOS CON LÍNEAS MEJORADAS POR SELECCIÓN GAMÉTICA Y RETROCRUZA1
}

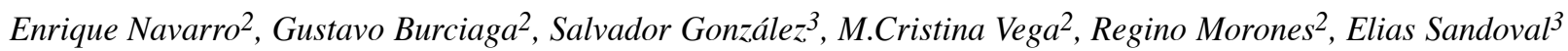

\section{RESUMEN}

Híbridos dobles de maíz formados con líneas mejoradas por selección gamética y retrocruza. El presente trabajo involucra la evaluación de 1040 híbridos dobles formados a partir de cruzas simples mejoradas a través del método de selección gamética y retrocruza, las mismas que fueron cruzadas con cinco probadores y asignadas a ocho experimentos, de acuerdo a la naturaleza de las cruzas simples. La evaluación se llevó a cabo en 1991 en Gómez Palacio, Durango, México, con los siguientes objetivos: 1) Evaluar el comportamiento de los híbridos dobles y 2) Determinar los caracteres agrónomicos de mayor importancia en la variación observada. Para rendimiento de grano, sobresalieron por su productividad los híbridos dobles formados con cruzas simples mejoradas por selección gamética, con rendimientos de hasta 11,6 t/ha, superando en un $23 \%$ al mejor del procedimiento de retrocruza, lo anterior es un reflejo del reducido por ciento de mazorcas podridas observado en selección gamética. Las cruzas simples mejoradas por retrocruzamiento fueron más precoces, de menor altura y con menos problemas de acame de raíz y tallo. Los mejores probadores fueron el 258-18-19 x MLS $^{4-1}$ y el 232 x 255-18-19, ya que al combinarse con algunas cruzas simples sus rendimientos de grano excedieron las 14 t/ha. Los máximos rendimientos de grano estuvieron asociados con valores altos de ACG en selección gamética. El análisis multivariado detectó que más de las dos terceras partes de la variación de los híbridos dobles es explicada por rendimiento de grano limpio, altura de mazorca y floración masculina-floración femenina.

\begin{abstract}
Double cross hibrids produced with lines improved by gamete and backcross selection. This research work includes theevaluation of 1040 double cross hybrids which were produced from single crosses improved by gamete and backcross procedures. Single hybrids were crossed with five testers. The yield trials were carried out during 1991 at Gómez Palacio, Durango, México, and the goals were as follows: 1) To evaluate the performance of double cross hybrids and 2) To determine the main agronomic traits on the observed variation. For grain yield, the double cross hybrids formed with single crosses improved by gamete selection performed better than those obtained by backross whose yields were adobe $11,6 \mathrm{t} / \mathrm{ha}$ ( $23 \%$ with respect to the best backcross genotype). The outstanding yields observed across the gamete procedure are probably due to the low percentage of rot ers. On the controry, the single crosses improved by backcross procedure showed early to flower, short height and had less problems ofboth root and stalk lodging. The best testers were AN258-18-19 x MLS ${ }^{4-1}$ and the AN 232 x 25518-19 when crossed with some single crosses yield more than $14 \mathrm{t} / \mathrm{ha}$. It is import to mention that the outstanding grain yields were associated with values of general combining ability with the gamete selection. Finally, the multivariate analysis showed that two-thirds of the observed variation in the double cross hybrids was given by grain yield and clean grain yield, plant and ear height, male and female flowering.
\end{abstract}

\section{INTRODUCCIÓN}

Los programas de mejoramiento genético de maíz dedican considerable tiempo y recursos económicos en la identificación de líneas progenitoras para la producción de híbridos comerciales. Sin embargo, la reducida base genética de la mayoría de las líneas así como diver- sos factores ambientales, son las principales causas para que las líneas sean susceptibles a plagas y enfermedades y a la falta de coincidencia en la floración entre otros; lo anterior se traduce en reducción del potencial de rendimiento de los híbridos. El mejoramiento genético continuo de las líneas no ha sido una práctica común en los programas de hibridación, ya que las líneas que confor-

\footnotetext{
1 Presentado en la XLII Reunión Anual del PCCMCA en El Salvador, Centroamérica, 1996.

2 Instituto Mexicano del Maíz (MM) y Departamento de Estadística y Cálculo. Universidad Autónoma Agraria Antonio Narro.25315. Saltillo, Coahuila, México.FAX (84) 177407.

3 Universidad de Guadalajara, Jalisco, México.
} 
man los híbridos fueron seleccionadas con base en su aptitud combinatoria y a su heterosis manifestada. Por otro lado, sería de gran utilidad comparar su versión mejorada, lo cual presumiblemente podría facilitar la producción de los híbridos e incluso superar la productividad de los mismos. Allard (1978) y Hallauer (1990) señalan la importancia del mejoramiento de líneas así como la utilidad de líneas recobradas por reciclaje de líneas.

El mejoramiento de líneas es una actividad muy importante dentro de un programa de mejoramiento genético, ya que permite mejorar los caracteres y corregir las deficiencias de los materiales existentes; sin perder la identidad de sus patrones heteróticos (Bauman, 1977).

Considerando lo anterior, el Instituto Mexicano del Maíz de la UAAAN ha venido desarrollando una serie de trabajos con el propósito de mejorar algunos caracteres de líneas progenitoras de híbridos para facilitar la producción de semilla híbrida y a la vez superar los rendimientos. El presente trabajo tuvo como objetivos: Evaluar el comportamiento de híbridos dobles y determinar los caracteres de mayor importancia en la variación observada.

\section{MATERIALES Y METODOS}

Se inició un programa de mejoramiento de líneas con base en los métodos de selección gamética (Stadler, 1944) y retrocruza (Harlan y Pope, 1922). Se seleccionaron fuentes de germoplasma con características de acuerdo al tipo de grano de las líneas involucradas con el propósito de mantener sus patrones heteróticos.

En el presente trabajo se generaron 1040 híbridos dobles formados a partir de cruzas simples recobradas o mejoradas por selección gamética y retrocruza, las mismas que fueron cruzadas con cinco probadores y agrupados en ocho experimentos de acuerdo a la naturaleza de sus cruzas simples. El diseño experimental fue en bloques al azar con dos repeticiones, se utilizaron tres testigos en cada uno de los ocho experimentos establecidos durante 1991 en Gómez Palacio, Durango, México. La parcela experimental consistió de un surco de 4,6 m de longitud espaciados a $0,8 \mathrm{~m}$, los cuales contenían 21 plantas a una distancia de $22 \mathrm{~cm}$.

Los caracteres medidos en cada parcela experimental fueron: días a floración, altura de planta y mazorca, acame de raíz y tallo, rendimiento de grano al $15,5 \%$ de humedad, entre otros. Para una mejor diferenciación y selección de las cruzas simples se estimó su aptitud combinatoria general, también se utilizó el análisis multivariado para cuantificar la variabilidad de los caracteres de importancia.

\section{RESULTADOS}

Información del comportamiento de las cruzas simples mejoradas para diferentes caracteres agronómicas se presenta en el Cuadro 1. Sobresalen por su productividad, los híbridos dobles formados por material mejorado a través de selección gamética con un promedio de 11,6 t/ha, superando los promedios obtenidos por retrocruza en los experimentos 2,3 y 4 (8,8; 10,5 y 9,0 $\mathrm{t} / \mathrm{ha}$, respectivamente) y al de los testigos (9,0 t/ha). Por otra parte es importante señalar que los híbridos dobles formados con material gen ético de gamética $\mathrm{x}$ retrocruza (experimentos $5 ; 6 ; 7$ y 8 ) tuvieron un promedio aceptable; ya que tuvieron un rendimiento de 10,4 t/ha (igual al promedio de los experimentos 2, 3 y 4, pero superior al observado en los testigos). El carácter días a floración tuvo una respuesta favorable en la metodología de retrocruza, ya que los híbridos mostraron valores inferiores en promedio (69 días) que aquellos observados por el procedimiento de gamética (71 días). La

Cuadro 1. Comportamiento agronómico de híbridos dobles formados con cruzas simples recobradas por selección gamética y retrocruza. Durango, México, 1991.

\begin{tabular}{lrrrrr}
\hline & \multicolumn{5}{c}{ Retrocruza gamética } \\
\cline { 2 - 6 } Caracter & Exp. 9 & Exp. 2 & Exp.3 & Exp. 4 & Testigos \\
\hline Días a flor & 71,0 & 67,0 & 69,0 & 71,0 & 69,0 \\
Altura de planta (cm) & 287,9 & 218,7 & 263,2 & 235,0 & 242,1 \\
Acame de raíz (\%) & 17,4 & 2,0 & 4,7 & 3,4 & 5,7 \\
Acame de tallo(\%) & 18,9 & 11,8 & 19,0 & 12,0 & 13,3 \\
Mazorcas podridas (\%) & 3,5 & 5,6 & 9,3 & 12,4 & 15,7 \\
Rendimiento de grano (t/ha- $\left.{ }^{-1}\right)$ & 11,6 & 8,8 & 10,5 & 9,0 & 9,0 \\
\hline
\end{tabular}


altura de planta siguió una tendencia similar que días a floración, ya que los híbrido s dobles de material de gamética tuvieron un porte alto $(287,9 \mathrm{~cm})$ versus al deretrocruzas $(218,7)$ y al de testigos $(242,1 \mathrm{~cm})$. El porcentaje de acame de raíz fue producido en aquellos materiales generados por retrocruza, principalmente los del experimento dos, no siendo así para los obtenidos por selección gamética $(17,4 \%)$ e inclusive éstos fueron superiores al de testigos $(5,7 \%)$. El porcentaje de acame de tallo tuvo una respuesta similar al acame de raíz, con la excepción deque los promedios deretrocruza al igual que de testigos fueron inferiores al mostrado por material de gamética. Finalmente, para el porcentaje de mazorcas podridas, sobresalió el promedio del material de gamético $(3,5 \%)$ en relación al de retrocruza (aunque con porcentajes inferiores al de testigos $15,7 \%$ ).

En el Cuadro 2, se pueden ver a las mejores cruzas simples mejoradas en combinación con varios probadores. Sobresalen las cruzas simples mejoradas por selección gamética cuyos rendimientos superaron las 15 t/ha en combinación con el probador 258-18-19, $\mathrm{x}$ MLS4-1 (49\% más que el mejor testigo experimental), con un intervalo de producción de 15,35 a 9,14 t/ha y una media de 12,05 t/ha. Por otro lado, el máximo rendimiento con el probador 232 x 255-18-19 fue del orden de 14,41 t/ha y la media alrededor de 11,16 t/ha. Por lo que respecta a la aptitud combinatoria general, ésta varió entre 3,41 a -2,46 y de 3,34 a -3,81 con un valor promedio de 2,78 y 2,75 para los probadores arriba mencionados; el valor más alto de ACG dentro de cada probador $(3,30$ y 3,26$)$ estuvo asociado con la máxima productividad (15,35 a 14,41 t/ha).

Dentro de las cruzas simples mejoradas a través de la metodología de retrocruzas destacan las combinadas con el probador 255-18-19 X ML 54-2 con rendimiento de $14,10 \mathrm{t} /$ ha $(37 \%$ superior en relación al mejor híbrido testigo), y un intervalo de productividad de 14,10 a 7,75. La productividad de las cruzas simples en combinación con los otros dos probadores se observó un rendimiento de (12,33 versus $11,17 \mathrm{t} / \mathrm{ha})$ para 232 10-11-1 x 255-18-19 y HAN 1 x HAN2, respectivamente. Al igual que en selección gamética, los valores más altos de ACG estuvieron asociados con los máximos rendimientos dentro de cada grupo.

Es de importancia señalar, que las cruzas simples mejoradas a través de selección gamética exhibieron los máximos rendimientos y en promedio los valores

Cuadro 2. Rendimiento y aptitud combinatoria general de las mejores cruzas simples mejoradas por selección gamética y retrocruza, en combinación con cinco probadores. Durange, México, 1991.

\begin{tabular}{lcc}
\hline Genotipo & Rendimiento de grano t/ha' & ACG \\
\hline C.S ${ }^{1} \times\left(258-18-19 \times \mathrm{MLS}_{4}-1\right)$ & 15,35 & 3,30 \\
C.S ${ }^{1} \times\left(258-18-19 \times \mathrm{MLS}_{4}-1\right)$ & 14,60 & 2,56 \\
C.S ${ }^{1} \times\left(258-18-19 \times \mathrm{MLS}_{4}-1\right)$ & 14,52 & 2,48 \\
C.S ${ }^{1} \times(232-10-11-1 \times 255-18-19)$ & 14,41 & 3,26 \\
C.S ${ }^{1} \times(232-10-11-1 \times 255-18-19)$ & 13,93 & 2,77 \\
C.S ${ }^{1} \times(232-10-11-1 \times 255-18-19)$ & 13,38 & 2,22 \\
C.S ${ }^{2} \times\left(255-18-19 \times \mathrm{MLS}_{4}-2\right)$ & 14,10 & 3,59 \\
C.S ${ }^{2} \times\left(255-18-19 \times \mathrm{MLS}_{4}-2\right)$ & 13,12 & 2,61 \\
C.S ${ }^{2} \times\left(255-18-19 \times \mathrm{MLS}_{4}-2\right)$ & 12,82 & 2,31 \\
C.S ${ }^{2} \times(232-10-11-1 \times 255-18-19)$ & 12,33 & 3,30 \\
C.S ${ }^{2} \times(232-10-11-1 \times 255-18-19)$ & 11,59 & 2,56 \\
C.S ${ }^{2} \times(232-10-11-1 \times 255-18-19)$ & 11,20 & 2,17 \\
$C^{2} . S^{2} \times\left(\mathrm{AN}_{1} \times \mathrm{AN}_{2}\right)$ & 11,17 & 2,25 \\
C.S ${ }^{2} \times\left(\mathrm{AN}_{1} \times \mathrm{AN}_{2}\right)$ & 10,64 & 1,71 \\
C.S ${ }^{2} \times\left(\mathrm{AN}_{1} \times \mathrm{AN}_{2}\right)$ & 10,64 & 1,61 \\
\hline
\end{tabular}

C. $S^{1}=$ cruza mejorada por selección gamética.

C. $S^{2}=$ cruza mejorada por retrocruza. 
más altos de ACG en relación aquellos bajo la metodología de retrocruza.

En el Cuadro 3 se presentan tres componentes principales, los cuales explican el 71,14\% de la variabilidad de los datos originales. El primer componente lo integran rendimiento de grano y rendimiento de grano limpio, éste último tiene una influencia mayor en dicho componente. El carácter mazorcas podridas mostró significancia $(\mathrm{P}<0,7)$, lo cual indica que algunos híbridos tienen capacidad de rendimiento con problemas de pudrición de mazorcas.

El segundo componente está constituido por los caracteres altura de planta y altura de mazorca con valores de 0,8979 y 0,8549 , respectivamente. El porcentaje de acame de raíz y tallo tuvieron valores altos $(0,6773$ y 0,6596$)$, sin embargo, fueron no significativas. El tercer componente está integrado por los caracteres días a floración masculina y días a floración femenina con valores significativos 0,9665 y 0,9646 , respectivamente.

\section{DISCUSION}

De los métodos de mejoramiento de líneas utilizados, el de selección gamética fue el más efectivo para caracteres tales como rendimiento de grano y porcentaje de mazorcas podridas con una superioridad de $10 \%$ y una reducción superior al $300 \%$; en relación aquellos materiales obtenidos por retrocruzas. Otros estudios confirman la eficiencia de la selección gamética en incrementar rendimiento de grano en maíz (Paredes, 1985; Bernal, 1990; y Ríos, 1993). Por otro lado, los materiales generados por retrocruzamiento fueron más precoces, de menor estatura y con menos problemas de acame de raíz y tallo. Lo anterior es contrario a lo reportado por Berlanga (1990), quien utilizando líneas recobrados por selección gamética observa mediana precocidad, poco daño de acame de raíz y tallo, aceptable altura de planta y mayor potencial de rendimiento y estabilidad de éste a través de ambientes.

Es de gran importancia seleccionar cruzas simples con excelente aptitud combinatoria, potencial de rendimiento, entre otros; ya que éstos serán los progenitores de los híbridos dobles (Ortega, 1990). En este estudio, se lograron identificar una serie de cruzas simples en combinación con varios probadores con valores altos de aptitud combinatoria y rendimiento de grano tanto para los materiales generados por selección gamética como los de retrocruza. Es importante señalar que con el primer procedimiento se tuvo una superioridad para rendimiento de grano de $9 \%$. En cuanto a la asociación de alta aptitud combinatorio con alto rendimiento, Oyervides (1979) observó en una serie de cruzas dialécticas que los progenitores de más alto ACG fueron también los que participaron en las mejores cruzas.

En cuanto a componentes principales se refiere, se observó que más del 70\% de la variabilidad existente en la evaluación de los 1040 híbridos dobles; se debió a la asociación de rendimiento de grano con rendimiento de grano limpio, altura de planta con altura de mazorca y

Cuadro 3. Componentes principales de los siguientes caracteres en la evaluación de 1040 híbridos dobles. Durango, México, 1991.

\begin{tabular}{|c|c|c|c|}
\hline Caracter & Componente $1^{* *}$ & Componente $2^{* * *}$ & Componente $3^{* * * *}$ \\
\hline Días a flor masculina & 0,049824 & $-0,065684$ & $0,966463^{*}$ \\
\hline Días a flor femenina & 0,054244 & $-0,051390$ & $0,964579^{*}$ \\
\hline Altura de planta $(\mathrm{cm})$ & 0,066935 & $0,897953^{*}$ & $-0,147421$ \\
\hline Altura de mazorca (cm) & 0,157469 & $0,854959^{*}$ & $-0,028667$ \\
\hline Acame de raíz (\%) & $-0,022028$ & 0,677262 & 0,119791 \\
\hline Acame de tallo (\%) & $-0,206997$ & 0,659655 & $-0,122460$ \\
\hline Mazorcas podridas (\%) & $-0,700957^{*}$ & 0,067236 & 0,181302 \\
\hline Mazorcas por cien plantas (\%) & 0,290649 & $-0,074190$ & 0,491983 \\
\hline Rendimiento de grano & $0,904844^{*}$ & 0,081591 & 0,153795 \\
\hline Rendimiento de grano limpio & $0,981583^{*}$ & 0,050202 & 0,074440 \\
\hline \multirow{3}{*}{\multicolumn{4}{|c|}{$\begin{array}{ll}* & \text { Valores marcados son }>0,7 \\
* * & \text { Rendimiento de grano total y limpio. } \\
* * * & \text { Altura de planta y de mazorca. }\end{array}$}} \\
\hline & & & \\
\hline & & & \\
\hline$* * * *$ Días a floración masculina y fe & & & \\
\hline
\end{tabular}


a la de floración masculina de mayor importancia en los programas de mejoramiento genético de maíz. No hay la menor duda en la utilidad de los análisis multi variados en seleccionar un número reducido de caracteres y así poder construir índices de selección que maximicen la superioridad genética en el menor tiempo posible.

\section{CONCLUSIONES}

La selección gamética produjo cruzas simples mejoradas más productivas que los obtenidos por retrocruza.

El procedimiento de retrocruza fue eficiente para caracteres tales como precocidad, altura de planta y acame de raíz y tallo.

El setenta porciento de la variabilidad en la evaluación de los híbridos dobles es presentada por la asociación, rendimiento de grano, rendimiento de grano limpio, altura de planta-altura de mazorca y floración masculina-floración femenina.

\section{LTERATURA CITADA}

ALLARD, R.W. 1978. Principios de la mejora gen ética de las plantas. Traducción al español por José L. Montoya. De. Omega, S.A. Barcelona, España.

BAUMAN, L.F. 1977. Improvement of established maize inbreds. Maydica XXII:213-222.
BERLANGA, P.S. 1990 Selección de líneas recobradas a partir de AN7 línea recobrada por el método de selección gamética. Tesis Licenciatura U.A.A.A.N., Buenavista, Saltillo, Coa., México.

BERNAL, F.J.C. 1990. Evaluación de cruzas de prueba de la línea AN 12 de maíz (Zea mays L.) recobradas por selección gamética. Tesis Licenciatura U.A.A.A.N., Buenavista, Saltillo, Coa. México.

HALLAUER, A.P. 1990. Methods used in developing maize inbreds. Maydica 35: 1-15.

HARLAN, H.V.; POPE, M.N. 1922. The use and value of backcrosses in small grain breeding. J. Hered. 13:319322.

ORTEGA, C.S. 1990. Selección de híbridos dobles e identificación de las mejores cruzas simples de maíz para la región del Bajío Mexicano. Tesis profesional UAAAN. Buenavista, Saltillo, Coa. México.

OYERVIDES, G.M. 1979. Estimación de parámetros genéticos, heterosis e índices de selección en variedades tropicales de maíz adaptadas a Nayarit. Tesis M.C. Chapingo, México.

PAREDES, M.R. 1985. Eficiencia de la selección gamética en el mejoramiento de dos líneas tropicales de maíz. III. Probador: línea tropical. tesis licenciatura. UAAAN. Buenavista, Saltillo, Coa., México.

RÍOS, B.V.M. 1993. Nuevos híbridos de maíz para el Bajío y Trópico seco obtenidos de líneas AN1 y AN2 recobradas por selección gamética. Tesis profesional UAAAN. Buenavista, Saltillo, Coa. México.

ST ADLER, L.J. 1944. Gamete selection in corn breeding. J. Am. Soc Agron 36:988-989. 